\title{
Stability of the glutathione supplementation into parenteral nutrition to protect very preterm babies from oxidative stress
}

\author{
Clémence Guiraut ${ }^{1}$, Nadine El Raichani ${ }^{1}$, Guillaume Morin ${ }^{1}$, Ibrahim Mohamed ${ }^{2}$ and \\ Jean-Claude Lavoie ${ }^{1, *}$ \\ 1 Department of Nutrition, Research Center of the CHU Sainte-Justine, Université de Montréal, Montréal, \\ Canada. Affiliation 1; clemence.guiraut@gmail.com; nadine.el.raichani@umontreal.ca, \\ guillaume.morin.6@umontreal.ca, jean-claude.lavoie@umontreal.ca \\ 2 Department of Paediatrics, Research Center of the CHU Sainte-Justine, Université de Montréal, Montréal, \\ Canada. Affiliation 2; Ibrahim.mohamed@umontreal.ca \\ * Correspondence: jean-claude.lavoie@umontreal.ca
}

\begin{abstract}
Most very premature newborns ( $<32$ weeks of gestation) receive parenteral nutrition $(\mathrm{PN})$ that is inherently contaminated with peroxides. Oxidative stress induced by $\mathrm{PN}$ is associated with bronchopulmonary dysplasia, a main pathological complication in these babies who have weak antioxidant capacity to detoxify peroxides because of their glutathione deficiency. In animals, glutathione supplementation of PN prevented oxidative stress and alveolar loss (the main characteristic of bronchopulmonary dysplasia). Of its two forms - disulfide (GSSG) and free thiol (GSH) - GSSG was used because of its better stability in PN. However, a 30\% loss of GSSG in PN is observed. The potentially high therapeutic benefits of GSSG supplementation on the health of very premature babies makes the study of its stability highly important. Thus, GSSG was incubated in combination with the following components of PN: dextrose, multivitamins, Primene, Travasol, as well as with cysteine, cystine and peroxides for $24 \mathrm{~h}$. Total glutathione in these solutions was measured 0-24h after the addition of GSSG. The combination of cysteine and multivitamins caused the maximum loss of glutathione. Removing the cysteine prevented the degradation of glutathione. GSSG reacts with cysteine to form cysteine-glutathione disulfide, another suitable glutathione substrate for preterm neonates.
\end{abstract}

Keywords: cysteine-glutathione disulfide; glutathione; glutathione supplementation; GSSG supplementation; oxidative stress; parenteral nutrition; premature newborns; pro-cysteine. 


\section{Introduction}

Very and extremely preterm babies, born within 32 weeks of gestation, account for about $2 \%$ of births in the United States [1]. The immaturity of their antioxidant capacity is considered to be a main factor making them vulnerable to several pathological complications such as bronchopulmonary dysplasia (BPD) [1-3]. The parenteral nutrition (PN) received by these newborns includes antioxidants such as ascorbate but is also contaminated with oxidant molecules such as peroxides (4-6). In fact, the multivitamin (MV) component of $\mathrm{PN}$ is the main source for the generation of peroxides. Under ambient light, riboflavin accelerates the reaction between ascorbate and dissolved oxygen to generate hydrogen peroxide (6-9). Thus, PN administered to these babies induces oxidative stress associated with the occurrence of bronchopulmonary dysplasia (BPD) $[3,10,11]$. BPD is the most common complication of extreme preterm birth and is a major public health concern $[3,12,13]$. BPD affects approximately 10,000 premature infants each year in the United States [12]. Although advances in the care of extremely and very preterm babies have been made, the incidence of BPD has not decreased and still leaves them with lifelong respiratory impairment $[12,14,15]$.

Glutathione is the primary endogenous antioxidant found in all eukaryotic cells and aims to counteract the intracellular effects of oxidative stress [16,17]. It detoxifies peroxides as a co-factor of glutathione peroxidases. Glutathione is a tri-peptide formed from the combination of glutamate, cysteine and glycine [18]. The enzymatic activity for its synthesis is mature in premature newborns [19]. The availability of cellular cysteine is well known to be a limiting step in the synthesis of glutathione [20]. However, cellular uptake of cysteine is immature in very preterm babies [18]. The levels of glutathione within cells of newborns is related to the duration of pregnancy; premature newborns show a decrease in glutathione stocks compared to term newborns [21-23]. The availability of glutathione to protect premature babies from the oxidative stress induced by peroxides that contaminated their PN seems to be an important player in the prevention of BPD [4.]. Very interestingly, in an animal model, glutathione supplementation of PN prevented oxidative stress and alveolar loss (typical of BPD) induced by PN contaminated with peroxides [4,24]. Therefore, it has been proposed to add glutathione in the PN of premature newborns to prevent oxidative stress and thus prevent the development of BPD. This added glutathione could play the role of pro-cysteine to improve the cellular synthesis of glutathione. The concept involves the action of $\gamma$-glutamyltansferase, which is mature in preterm newborns [19]. This enzyme transfers the $\gamma$-glutamyl moiety of glutathione to a circulating amino acid. The remaining cysteinylglycine dipeptide, as well as the new $\gamma$-glutamyl-amino acid dipeptide, are taken-up by cells where dipeptidases release cysteine for a new glutathione synthesis $[25,26$.$] . This mechanism is a$ physiological pathway for enriching cells with cysteine [27].

Glutathione is available into two forms with equal in vivo affinity for $\gamma$-glutamyltansferase [28]: oxidized (GSSG) and reduced (GSH). In animals, GSSG has been used to supplement PN because it has a better stability in solution [29]. However, a loss of GSSG of approximately $30 \%$ was still observed after $24 \mathrm{~h}$ in the PN [29]. In addition to a possible decrease in the effectiveness of glutathione supplementation, this loss highlights the need to identify potential by-products that could also limit the relevance of this supplementation. The very promising therapeutic benefits of GSSG supplementation on the health of premature babies make the study of its stability of great importance. The aim of this work was to study the interactions of GSSG with the components of PN in order to understand this loss and to identify the new compound(s) possibly formed.

\section{Materials and Methods}

\subsection{Solutions of parenteral nutrition}

All solutions contained the oxidized form of L-glutathione $50 \mu \mathrm{M}$ (GSSG, Sigma \#G4376, Oakville, ON, Canada). The parenteral nutrition (PN) of reference contained Dextrose 10\% (w,v) (Dextrose 70\%, 
Baxter, Mississauga, ON, Canada), amino acid preparation Primène 2\% ( $\mathrm{w}, \mathrm{v})$ (Primene 10\%; Baxter, Mississauga, ON, Canada), multivitamin preparation 2\% (v,v) (Multi-12 pediatric; Sandoz, Montreal, QC, Canada). When needed, Primene was replaced by nanopure water, amino acid preparation Travasol (Travasol 10\%; Baxter, Mississauga, ON, Canada), L-cysteine $3.35 \mathrm{mM}$ (Sigma \#C8152, Oakville, ON, Canada) or L-cystine 1.675mM (Sigma \#C7602, Oakville, ON, Canada). The PN solution was supplemented with hydrogen peroxide 0-500 $\mu \mathrm{M}\left(\mathrm{H}_{2} \mathrm{O}_{2}\right.$, Sigma \#216763, Oakville, ON, Canada) to study the effect of peroxides onto glutathione stability. The PN were incubated at room temperature $\left(22.7 \pm 0.2^{\circ} \mathrm{C}\right)$ for up to $24 \mathrm{~h}$ under exposure to $0-200$ foot-candles (FC) light intensities. Light intensities were measured using a light meter (Fisher \#S90198, Ottawa, ON, Canada). The 0 FC intensity was obtained by manipulating solutions in an unilluminated room in which the real light intensity was 0.3 FC for the duration of the manipulations ( $2 \times 15$ min among the $24 \mathrm{~h}$ of incubation). All the solutions were placed on dry ice immediately after sampling and kept at $-80^{\circ} \mathrm{C}$ until analysis. All the experiments were performed 3 times and each PN was measure 3 times for each parameter except for peroxides, which were measured once per PN.

\subsection{Glutathione stability}

The two glutathione species (oxidized glutathione: GSSG or reduced glutathione: GSH), referred as glutathione, were measured with an enzymatic assay [31]. This assay was based on the recycling of GSSG into GSH by the glutathione reductase in the presence of 2,2'-Dinitro-5,5'-dithiobenzoic acid 590 $\mu \mathrm{M}$ (DTNB, Sigma \#D8130, Oakville, ON, Canada). This reaction generates 2-nitro-5-thiobenzoate anion (TNB) over time that was read at $412 \mathrm{~nm}$ as previously described. This method measured total glutathione (GSH + GSSG) that was reported as GSH equivalent.

\subsection{Peroxides concentrations}

The ferrous oxidation-xylenol orange (FOX) method [32] was used to assess peroxide concentrations in PN. PN were diluted ex temporani in nanopure water and incubated for $30 \mathrm{~min}$ in FOX reagent. FOX reagent consisted of $\mathrm{H}_{2} \mathrm{SO}_{4} 50 \mathrm{mM}$ (Baker \#9681, Center Valley, PA, USA), xylenol orange $114 \mu \mathrm{M}$ (Sigma \#227854, Oakville, ON, Canada), FeCl $252 \mu \mathrm{M}$ (Sigma \#220299, Oakville, ON, Canada) and 2,6-Di-tert-butyl-4-methylphenol $4 \mathrm{mM}$ (BHT, Sigma \#240028, Oakville, ON, Canada) in methanol (ACP \#M-3640, Montreal, QC, Canada). Solutions were then centrifuged at 7,200g for $1 \mathrm{~min}$ and the supernatant's absorbance was read at $560 \mathrm{~nm}$ and compared to a $\mathrm{H}_{2} \mathrm{O}_{2}$ standard curve.

\subsection{Cysteine concentration}

Cysteine was measured in solution by reducing DTNB $590 \mu \mathrm{M}$ (Sigma \#D8130, Oakville, ON, Canada) into TNB whose yellow color is measured at $412 \mathrm{~nm}$. Cysteine concentration was extrapolated using the DTNB molar extinction coefficient of $14150 \mathrm{M}^{-1} \mathrm{~cm}^{-1}$.

\subsection{Cysteine-glutathione disulfide determinations}

LC-MS analyses were performed to identify cysteine-glutathione disulfide within the PN. The LC-MS analyses were performed on an Agilent Technologies 6224 TOF coupled to a 1260 series liquid chromatography system. The column used was a Synergi Hydro-RP from Phenomenex $(150 \times 4.6 \mathrm{~mm}$, $4 \mu \mathrm{m}$ ) with eluent $\mathrm{A}, 20 \mathrm{mM}$ ammonium acetate with $0.1 \%$ formic acid and eluent B, methanol. The gradient started at $0 \% \mathrm{~B}$ and kept isocratic for $1.5 \mathrm{~min}$ and then increased to $60 \% \mathrm{~B}$ in $11.5 \mathrm{~min}$ for a total run time of $20 \mathrm{~min}$. Data were acquired in positive electrospray, scanning from $\mathrm{m} / \mathrm{z} 100$ to 3000 with dual spray allowing for accurate mass measurement. For empirical formula confirmation a mass tolerance of $5 \mathrm{mDa}$ was used. The assay was performed by the Centre regional de spectrométrie de masse, Université de Montréal. 


\subsection{Statistics}

Glutathione concentrations between groups after 24h of incubation in Figure 1, Tables 1 and 2 were compared by ANOVA. Glutathione and cysteine concentration between $0 \mathrm{~h}$ and $24 \mathrm{~h}$ for each group in Tables 3 and 4 were analyzed with the student $t$-test. Data are presented as mean \pm SEM. The threshold of significance was set at $\mathrm{p}<0.05$.

\section{Results}

\subsection{Glutathione loss in according to PN components}

Oxidized glutathione (GSSG) was first incubated under ambient light (259 $\pm 12,5$ FC) with a single or combined component of PN namely dextrose $10 \%(\mathrm{D})$, multivitamins $2 \%(\mathrm{MV})$, Primène $2 \%$ (amino acids) (Figure 1). The composition of this hydrophilic part of the PN is similar to that usually received by a premature newborn. After $24 \mathrm{hr}$ incubation, $\mathrm{D} \pm \mathrm{MV}$ did not induce the degradation of glutathione. D + Primene induced a $19 \pm 3 \%$ loss of glutathione. As previously observed [21], the complete PN containing D + Primene + MV showed a $33 \pm 3 \%$ loss of glutathione. Given that the glutathione loss was maximal at $24 \mathrm{~h}$, we collected the data at $0 \mathrm{~h}$ and $24 \mathrm{~h}$ timepoints for the following experiments.

Figure 1. Degradation of glutathione in PN exposed to ambient light (259 $\pm 12,5$ FC). The PN containing Dextrose + Primene + MV showed the most important loss of glutathione after 24h. D: dextrose, MV: multivitamins, ns: non significant, ${ }^{* *}: \mathrm{p}<0.01,{ }^{* * *}: \mathrm{p}<0.001$.

Exposure to light of MV solutions or PN solutions that contain MV promote the generation

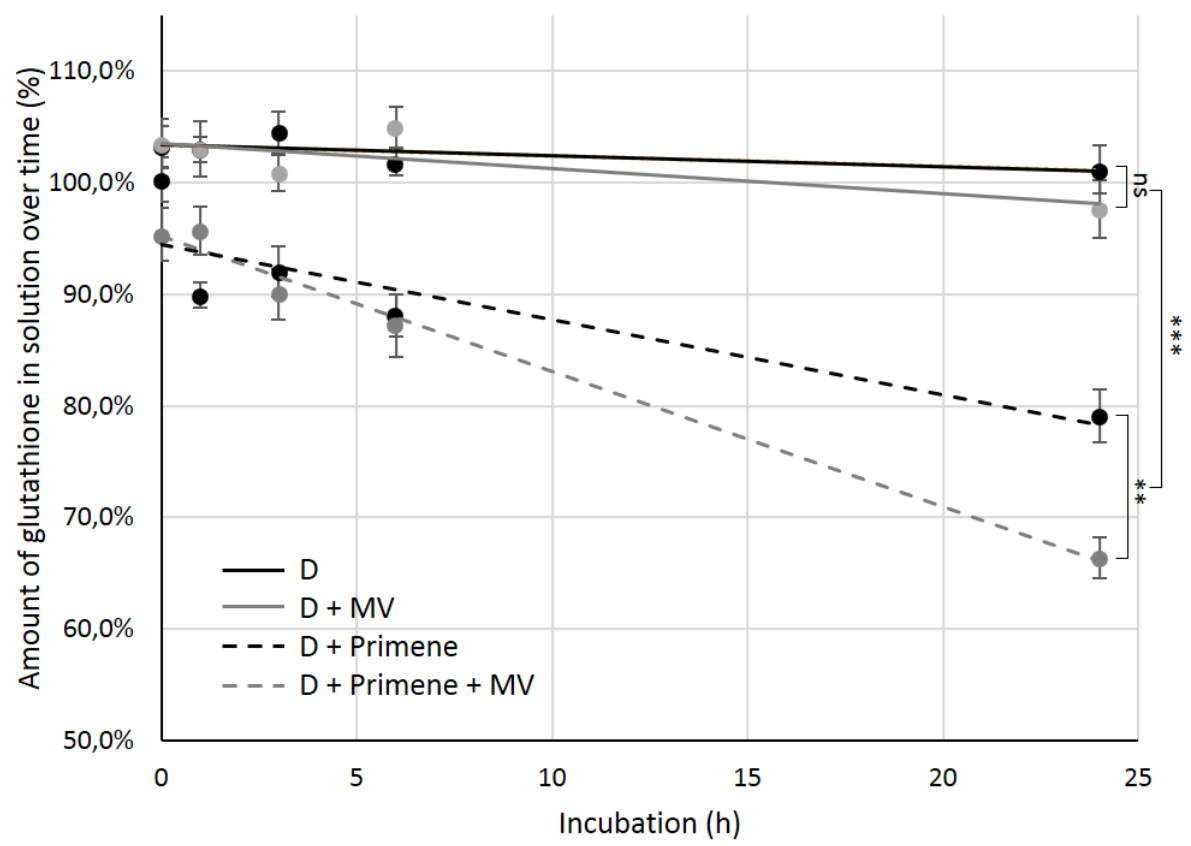

of peroxides [6]. The additional effect of MV on Primene prompted us to assess the effects of exposure to light on glutathione levels.

\subsection{Increased loss of glutathione within PN exposed to increasing light intensities}


PN containing D 10\% + Primene 2\% + MV 2\% were incubated under light intensities varying from $0 \mathrm{FC}$ (no light) to $200 \mathrm{FC}$ for $24 \mathrm{~h}$. The maximum light intensity measured in the neonatal intensive care unit of CHU Sainte-Justine was $150 \mathrm{FC}$. The increase in light intensities induced an increased loss $(\mathrm{p}<0.01)$ of glutathione (Table 1). While the loss of glutathione was $16 \pm 1 \%$ at $0 \mathrm{FC}$, it doubled at $200 \mathrm{FC}(\mathrm{p}<0.01)$. This observation led us to incubate the complete PN with increasing concentrations of peroxides because light-exposed PN containing MV mainly generates peroxides [6].

Table 1. Impact of the light exposure of PN solution on glutathione degradation.

\begin{tabular}{lc}
\hline PN components & $\begin{array}{c}\text { Loss of glutathione after } \\
\text { 24h of incubation (\%) }\end{array}$ \\
\hline D + Primene + MV - Light (0 FC) & $16 \pm 1$ \\
D + Primene + MV + Light (40 FC) & $25 \pm 1$ \\
D + Primene + MV + Light (200 FC) & $32 \pm 2$ \\
\hline
\end{tabular}

\subsection{MV-related loss of glutathione in PN is not caused by peroxides}

PN containing D 10\% + Primene $2 \%$ were incubated 24h with increasing concentrations of peroxides ranging from $0 \mu \mathrm{M}$ to $500 \mu \mathrm{M} \mathrm{H}_{2} \mathrm{O}_{2}$ to mimic increasing concentrations observed with MV. Concentrations of 150, 300 and $500 \mu \mathrm{M} \mathrm{H}_{2} \mathrm{O}_{2}$ mimicked those obtained with 1, 2 and $3 \% \mathrm{MV}$, respectively. The addition of increasing peroxide concentrations did not affect glutathione levels in the PN (Table 2). This meant that the loss of glutathione was not caused per se by the peroxides generated by MV. Peroxides might not be the main trigger for loss of glutathione. Given the interaction between Primene and MV observed in Figure 1, we hypothesized that a component of Primene could act in concert with MV to destabilize the GSSG structure.

Table 2. Impact of peroxides into PN solution on glutathione degradation

\begin{tabular}{lc}
\hline PN components & $\begin{array}{c}\text { Loss of glutathione after } \\
\text { 24h of incubation }(\%)\end{array}$ \\
\hline $\mathrm{D}+$ Primene $-\mathrm{H}_{2} \mathrm{O}_{2}$ & $18 \pm 1$ \\
$\mathrm{D}+$ Primene $+\mathrm{H}_{2} \mathrm{O}_{2}(150 \mu \mathrm{M})$ & $20 \pm 3$ \\
$\mathrm{D}+$ Primene $+\mathrm{H}_{2} \mathrm{O}_{2}(300 \mu \mathrm{M})$ & $15 \pm 1$ \\
$\mathrm{D}+$ Primene $+\mathrm{H}_{2} \mathrm{O}_{2}(500 \mu \mathrm{M})$ & $22 \pm 3$ \\
\hline
\end{tabular}

\subsection{Synergistic impact of cysteine and $M V$ on glutathione loss in $P N$}

Given that cysteine and cystine respectively have a free thiol group and a disulfide bridge that are available to react with the disulfide bond of GSSG, we hypothesized that they are the main contributors to the reaction leading to loss of glutathione. Primene 2\% (amino acid solution containing cysteine, part of which is oxidized to cystine) was replaced by i) Travasol $2 \%$ (amino acid solution without cysteine or cystine), ii) Travasol $2 \%$ + cysteine $3.35 \mathrm{mM}$ (equivalent to the total cysteine content (if not oxidized) of Primene), iii) Travasol 2\% + cystine $1.675 \mathrm{mM}$ (if all of Primene's cysteine is oxidized), iv) sole cysteine $3.35 \mathrm{mM}$ or v) sole cystine $1.675 \mathrm{mM}$. These solutions were incubated with or without MV $2 \%$ to thoroughly study the interaction between amino acids and MV (Table 3). As previously shown in Figure 1, the addition of MV to D + Primene doubled the degradation of glutathione. The removal of cysteine from PN by replacing Primene with Travasol protected the GSSG from degradation regardless of the presence of MV. Adding MV to Travasol+cysteine or to the cysteine alone resulted in the greatest loss of glutathione. These results 
confirms that in $\mathrm{PN}$, cysteine, and not cystine, had a triggering role in the loss of glutathione and that its action was in concert with the action of MV.

Table 3. Impact of cysteine and MV on glutathione degradation

\begin{tabular}{lcc}
\hline & \multicolumn{2}{c}{ Loss of glutathione after 24h incubation } \\
\cline { 2 - 3 } PN components & without MV & with MV \\
\hline D + Primene & $19 \pm 4^{*}$ & $43 \pm 2^{* *}$ \\
D + Travasol (No cysteine) & $0 \pm 4$ & $-2 \pm 4$ \\
D + Travasol + cysteine & $8 \pm 1^{*}$ & $76 \pm 2^{* * *}$ \\
D + Travasol + cystine & $1 \pm 2$ & $-1 \pm 3$ \\
Cysteine & $8 \pm 1^{*}$ & $64 \pm 4^{* *}$ \\
Cystine & $0 \pm 2$ & $11 \pm 2^{*}$ \\
\hline \multicolumn{2}{c}{${ }^{*}<0.05^{* *} \mathrm{p}<0.01, * * \mathrm{p}<0.001$ between $0 \mathrm{~h}$ and $24 \mathrm{~h}$ incubation. }
\end{tabular}

\subsection{Specific role of cysteine in the glutathione loss within PN}

In addition to glutathione, cysteine was measured in the solutions described in 3.4. to understand if cysteine had a causal role in the loss of glutathione. The addition of MV to D + Primene significantly reduced the concentration of cysteine remaining in solution after $24 \mathrm{~h}$ from $88 \pm 5 \%$ without MV to $24 \pm 1 \%$ with MV. The addition of MV to cysteine alone (+ D) or in combination with Travasol induced the total loss of this amino acid in 24 hours, in parallel with the loss of glutathione observed in Table 3. This last result suggests that cysteine played an active role in the loss of glutathione. Cysteine could act in concert with MV to disrupt the disulfide bond of GSSG. As expected, no significant concentration of cysteine concentrations was detected when cystine was added to the PN instead of cysteine.

Table 4. Impact of cysteine and MV on cysteine consumption in PN supplemented with GSSG

\begin{tabular}{lccc}
\hline & & \multicolumn{2}{c}{ Amounts of cysteine, mM (\%) } \\
\cline { 3 - 4 } PN components & Incubation $(\mathrm{h})$ & without MV & with MV \\
\hline D + Primène & 0 & $2.05 \pm 0.05(100 \pm 2)$ & $2.16 \pm 0.09(100 \pm 4)$ \\
& 24 & $1.80 \pm 0.11(88 \pm 5)$ & $0.52 \pm 0.03(24 \pm 1)^{* *}$ \\
D + Travasol + cysteine & 0 & $3.53 \pm 0.08(100 \pm 2)$ & $3.57 \pm 0.30(100 \pm 8)$ \\
& 24 & $1.92 \pm 0.04(54 \pm 1)^{* * *}$ & $0.13 \pm 0.02(4 \pm 1)^{* *}$ \\
Cysteine & 0 & $3.69 \pm 0.04(100 \pm 1)$ & $3.27 \pm 0.05(100 \pm 2)$ \\
& 24 & $3.62 \pm 0.03(98 \pm 1)^{*}$ & $0.04 \pm 0.02(1 \pm 1)^{* * *}$ \\
Cystine & 0 & 0 & $0.02 \pm 0.02$ \\
& 24 & 0 & $0.02 \pm 0.01$ \\
\hline
\end{tabular}

${ }^{*} p<0.05,{ }^{* *} p<0.01,{ }^{* * *} p<0.001$ between $0 h$ and $24 h$ for each solution 
The apparent loss of glutathione in the presence of cysteine prompted us to perform further analyses to identify the most likely compound, cysteine-glutathione disulfide (CYSSG), which could be created by the reaction between glutathione and cysteine. Thus, we performed LC-MS analyses to identify CYSSG at $0 \mathrm{~h}$ and $24 \mathrm{~h}$ in solutions containing Cysteine $+\mathrm{MV} \pm$ GSSG . A peak corresponding to CYSSG (m/z 427.09518) appeared after $24 \mathrm{~h}$ in the solution containing Cysteine + MV + GSSG

(Figure 2). This peak was not detected in the solution containing Cysteine + MV - GSSG (data not shown).

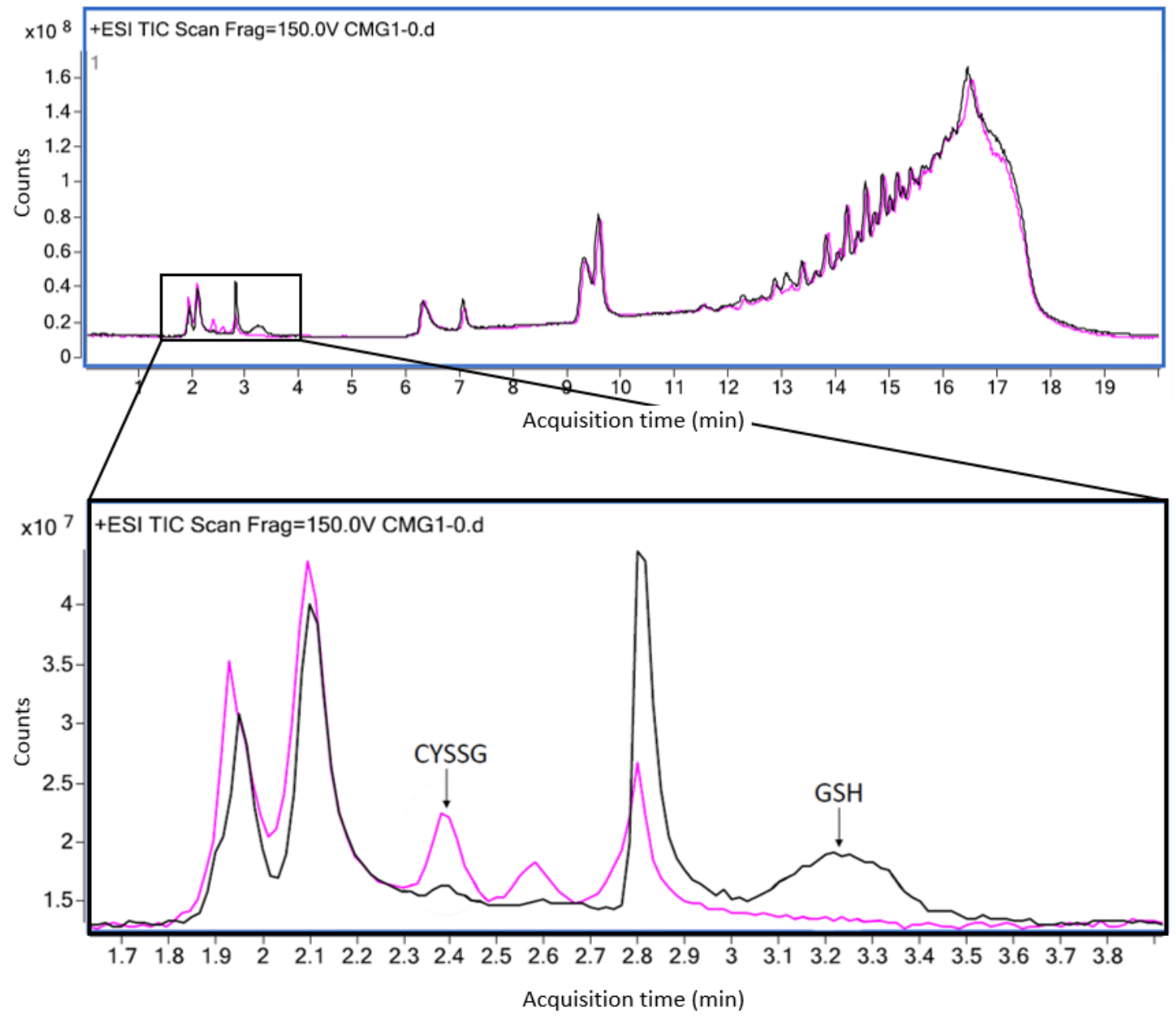

Figure 2. Total ion chromatograph of solutions containing Cysteine + MV + GSSG after 0 or 24h. A peak of CYSSG appears after 24h. Black: 0h, Pink: 24h. CYSSG : cysteine-glutathione disulfide, GSH: reduced glutathione.

\section{Discussion}

PN supplementation with glutathione is intended to be used as a pro-cysteine in vivo to support the synthesis of glutathione, particularly in premature newborns who have a low level of this endogenous antioxidant molecule [4]. However, part of the glutathione used as a supplement disappeared from the PN solution [29]. This in vitro study aimed to elucidate the mechanisms causing this loss of glutathione in a PN solution whose composition was similar to that administered to premature infants. The loss observed here was comparable to what had previously been reported in a similar PN solution [29]. 
The study demonstrates that the presence of cysteine is crucial for the loss of glutathione in PN. Because this loss was mirrored by a loss of cysteine, we suspected a reaction between the two molecules. The interactions between the free thiol of GSH or the disulfide bond of GSSG with other thiol-containing molecules such as cysteine and cystine are well known [33,34]. Since cystine was ineffective, a reaction between GSSG and cysteine was suspected. This reaction can generate cystine, GSH as well as cysteine-glutathione disulfide (CySSG). The generation of GSH cannot be detected by our assay, because the method used for the titration of glutathione detected the total of the two forms of glutathione (GSH and GSSG). In addition, if the formation of GSH was real, it would not be detrimental as GSH is also used by $\gamma$-glutamyltransferase to enrich the body with cysteine. The production of cysteine-glutathione disulfide was confirmed by the LC-MS technology. This is also a favourable outcome since this molecule is normally present in human plasma [35] and is recognized as being pro-glutathione [30].

Because removal of MV or photoprotection of PN only partially prevents loss of glutathione, we suspected that the spontaneous reaction between cysteine and GSSG is accelerated by the photo-excited riboflavin that could transfer the energy from the light to the reaction (Figure 3 ). This hypothesis is based on a study reporting occurrence of such a reaction between ascorbate and dissolved oxygen during the generation of hydrogen peroxide in PN. This reaction is accelerated by the photo-exited riboflavin present in MV [36]. Acceptance of this concept implied a possible role for peroxides. However, the lack of effect observed by adding peroxides has excluded this possibility.

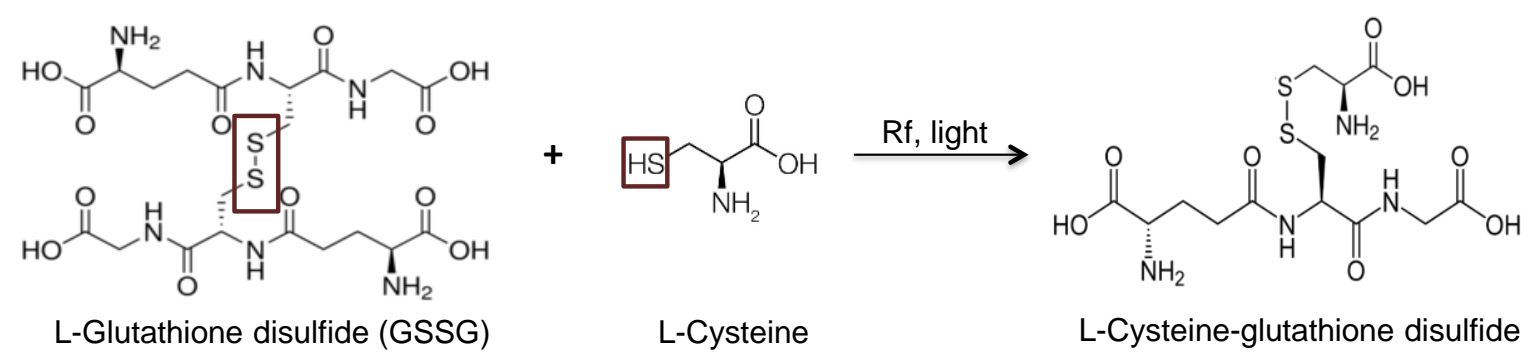

Figure 3. In the presence of cysteine, transformation of L-glutathione disulfide into L-cysteine-glutathione disulfide. A reaction favored both by exposure to ambient light and to the parenteral preparation of multivitamin, which contains the photosensitive riboflavin (Rf).

The aim of this work was to study the interactions of GSSG with the components of PN in order to understand this loss and to identify the new compound(s) possibly formed. The study clearly demonstrated that the loss of glutathione in PN is caused by the reaction between GSSG and cysteine leading to the formation of a mixed disulfide called cysteine-glutathione disulfide. This molecule is formed endogenously in mammals. It is present in human plasma [30] where it is considered to be pro-glutathione [30].

In conclusion, the study validates the safety of supplementing PN with GSSG to act as an effective pro-cysteine in vivo. As previously discussed [4], this supplement would be advantageous compared to the use other precursors such as $\mathrm{N}$-acetylcysteine. Cochrane meta-analysis concluded that adding cysteine or $\mathrm{N}$-acetylcysteine to $\mathrm{PN}$ is ineffective in improving glutathione levels in premature babies or reducing the incidence of BPD [37].

Author Contributions: Conceptualization, Clémence Guiraut and Jean-Claude Lavoie; Data curation, Clémence Guiraut, Nadine El Raichani and Guillaume Morin; Funding acquisition, Ibrahim Mohamed and Jean-Claude Lavoie; Methodology, Clémence Guiraut, Nadine El Raichani and Jean-Claude Lavoie; Supervision, Jean-Claude Lavoie; Writing - original draft, Clémence 
Guiraut and Jean-Claude Lavoie; Writing - review \& editing, Clémence Guiraut, Nadine El Raichani, Guillaume Morin, Ibrahim Mohamed and Jean-Claude Lavoie.

Funding: This research was funded by the Canadian Institutes of Health Research, grant number PJT-148522.

Acknowledgments: We thank the Mass Spectrometry platform from the Department of Chemistry of the Université de Montréal for its help in the identification of cysteine-glutathione disulfide.

Conflicts of Interest: The authors declare no conflict of interest. 


\section{References}

1. Glass, H.C.; Costarino, A.T.; Stayer, S.A.; Brett, C.M.; Cladis, F.; Davis, P.J. Outcomes for extremely premature infants. Anesth. Analg. 2015, 120, 1337-1351.

2. Patel, P.; Bhatia, J. Total parenteral nutrition for the very low birth weight infant. Semin Fetal Neonatal Med 2017, 22, 2-7.

3. Perrone, S.; Tataranno, M.L.; Buonocore, G. Oxidative stress and bronchopulmonary dysplasia. J Clin Neonatol 2012, 1, 109-114.

4. Lavoie, J.C.; Chessex, P. Parenteral nutrition and oxidant stress in the newborn: A narrative review. Free Radic Biol Med 2019, 142, 155-167.

5. Bassiouny, M.R.; Almarsafawy, H.; Abdel-Hady, H.; Nasef, N.; Hammad, T.A.; Aly, H. A randomized controlled trial on parenteral nutrition, oxidative stress, and chronic lung diseases in preterm infants. J. Pediatr. Gastroenterol. Nutr. 2009, 48, 363-369.

6. Lavoie, J.C.; Bélanger, S.; Spalinger, M.; Chessex, P. Admixture of a multivitamin preparation to parenteral nutrition: the major contributor to in vitro generation of peroxides. Pediatrics 1997, 99, E6.

7. Laborie, S.; Lavoie, J.C.; Pineault, M.; Chessex, P. Contribution of multivitamins as well as air and light in the generation of peroxides in adult and neonatal parenteral nutrition solutions. Ann Pharmacother 2000, 34, 440-445.

8. Laborie, S.; Lavoie, J.C.; Chessex, P. Increased urinary peroxides in newborn infants receiving parenteral nutrition exposed to light. J Pediatr 2000, 136, 628-632.

9. Laborie, S.; Lavoie, J.C.; Chessex, P. Paradoxical role of ascorbic acid and riboflavin in solutions of total parenteral nutrition: Implication in photoinduced peroxide generation. Pediatr Res 1998, 43, 601-606.

10. Mohamed, I.; Elremaly, W.; Rouleau, T.; Lavoie, J.-C. Oxygen and parenteral nutrition two main oxidants for extremely preterm infants: "It all adds up." J Neonatal Perinatal Med 2015, 8, 189-197.

11. Wang, J.; Dong, W. Oxidative stress and bronchopulmonary dysplasia. Gene 2018, 678, 177-183.

12. McEvoy, C.T.; Jain, L.; Schmidt, B.; Abman, S.; Bancalari, E.; Aschner, J.L. Bronchopulmonary dysplasia: NHLBI Workshop on the Primary Prevention of Chronic Lung Diseases. Ann Am Thorac Soc 2014, 11 Suppl 3, S146-153.

13. Stoll, B.J.; Hansen, N.I.; Bell, E.F.; Shankaran, S.; Laptook, A.R.; Walsh, M.C.; Hale, E.C.; Newman, N.S.; Schibler, K.; Carlo, W.A.; et al. Neonatal outcomes of extremely preterm infants from the NICHD Neonatal Research Network. Pediatrics 2010, 126, 443-456.

14. Doyle, L.W.; Victorian Infant Collaborative Study Group Respiratory function at age 8-9 years in extremely low birthweight/very preterm children born in Victoria in 1991-1992. Pediatr. Pulmonol. 2006, 41, 570-576.

15. Vom Hove, M.; Prenzel, F.; Uhlig, H.H.; Robel-Tillig, E. Pulmonary outcome in former preterm, very low birth weight children with bronchopulmonary dysplasia: a case-control follow-up at school age. J. Pediatr. 2014, 164, 40-45.e4.

16. Stone, W.L.; Basit, H.; Mohiuddin, S.S. Biochemistry, Antioxidants. In StatPearls; StatPearls Publishing: Treasure Island (FL), 2019. 
17. Gould, R.L.; Pazdro, R. Impact of Supplementary Amino Acids, Micronutrients, and Overall Diet on Glutathione Homeostasis. Nutrients 2019, 11.

18. Lavoie, J.-C.; Rouleau, T.; Truttmann, A.C.; Chessex, P. Postnatal gender-dependent maturation of cellular cysteine uptake. Free Radic. Res. 2002, 36, 811-817.

19. Lavoie, J.C.; Chessex, P. Development of glutathione synthesis and gamma-glutamyltranspeptidase activities in tissues from newborn infants. Free Radic. Biol. Med. 1998, 24, 994-1001.

20. Wu, G.; Fang, Y.Z.; Yang, S.; Lupton, J.R.; Turner, N.D. Glutathione metabolism and its implications for health. J Nutr 2004, 134, 489-492.

21. Lavoie, J.C.; Chessex, P. Gender and maturation affect glutathione status in human neonatal tissues. Free Radic. Biol. Med. 1997, 23, 648-657.

22. Lavoie, J.-C.; Rebeuh, J.; Herzog, D.; Faure, C.; Rouleau, T. Oxidative Stress in Children on Long-Term Parenteral Nutrition. J Hum Nutr Food Sci 2017, 5, 1110.

23. Mohamed, I.; Elremaly, W.; Rouleau, T.; Lavoie, J.-C. Ascorbylperoxide Contaminating Parenteral Nutrition Is Associated With Bronchopulmonary Dysplasia or Death in Extremely Preterm Infants. JPEN J Parenter Enteral Nutr 2017, 41, 1023-1029.

24. Elremaly, W.; Mohamed, I.; Rouleau, T.; Lavoie, J.-C. Adding glutathione to parenteral nutrition prevents alveolar loss in newborn Guinea pig. Free Radic. Biol. Med. 2015, 87, 274-281.

25. Cotgreave, I.A.; Schuppe-Koistinen, I. A role for gamma-glutamyl transpeptidase in the transport of cystine into human endothelial cells: relationship to intracellular glutathione. Biochim Biophys Acta 1994, 1222, 375-382.

26. Deneke, S.M.; Susanto, I.; Vogel, K.A.; Williams, C.E.; Lawrence, R.A. Mechanisms of use of extracellular glutathione by lung epithelial cells and pulmonary artery endothelial cells. $A m \mathrm{~J}$ Respir Cell Mol Biol 1995, 12, 662-668.

27. Meister, A.; Anderson, M.E. Glutathione. Ann Rev Biochem 1983, 52, 711-760.

28. McIntyre, T.M.; Curthoys, N.P. Comparison of the hydrolytic and transfer activities of rat renal gamma-glutamyltranspeptidase. J Biol Chem 1979, 254, 6499-6504.

29. Elremaly, W.; Mohamed, I.; Rouleau, T.; Lavoie, J.-C. Impact of glutathione supplementation of parenteral nutrition on hepatic methionine adenosyltransferase activity. Redox Biol 2016, 8, 18-23.

30. Berkeley, L.I.; Cohen, J.F.; Crankshaw, D.L.; Shirota, F.N.; Nagasawa, H.T. Hepatoprotection by L-cysteine-glutathione mixed disulfide, a sulfhydryl-modified prodrug of glutathione. J Biochem Mol Toxicol 2003, 17, 95-97.

31. Griffith, O.W. Determination of glutathione and glutathione disulfide using glutathione reductase and 2-vinylpyridine. Anal Biochem 1980, 106, 207-212.

32. Jiang, X.Y.; Wooland, A.C.S.; Wolf, S.P. Lipid hydroperoxides measurement by oxidation of $\mathrm{Fe} 2+$ in the presence of xylenol orange. Comparison with the TBA assay and an iodometric method. Lipids 1991, 26, 853-856.

33. Jones, D.P. Radical-free biology of oxidative stress. Am J Physiol Cell Physiol 2008, 295, C849-C868.

34. Yi, M.C.; Khosla, C. Thiol-disulfide exchange reactions in the mammalian extracellular environment. Annu Rev Chem Biomol Eng 2016, 7, 197-222. 
35. Kleinman, W.A.; Richie, J.P. Jr. Status of glutathione and other thiols and disulfides in human plasma. Biochem Pharmacol 2000, 60, 19-29.

36. Laborie, S.; Lavoie, J.C.; Chessex, P. Paradoxical Role of Ascorbic Acid and Riboflavin in Solutions of Total Parenteral Nutrition: Implication in Photoinduced Peroxide Generation. Pediatr Res 1998, 43, 601-606.

37. Soghier, L.M. Brion, L.P. Cysteine, cystine or N-acetylcysteine supplementation in parenterally fed neonates, Cochrane Database Syst Rev 2006, 18, CD004869. 